\title{
Abscesos renales y peri-renales: análisis de 44 casos
}

\author{
Juan Fullá O., Oscar Storme C., Alberto Fica C., M. Antonieta Varas P., \\ José Flores M., Fernando Marchant G. y Daniel Varas F.
}

\section{Renal and perinephric abscesses: a series of 44 cases}

Renal abscesses are infrequent event and may occasionally be fatal. In order to characterize its main clinical features, its diagnosis and evolution, a retrospective-descriptive study was done with cases identified between 1996 and 2006 in a teaching hospital. Forty-four cases were collected (mean age 49.9 years). Diabetes mellitus was present in $38.6 \%$, urinary calculi in $36.4 \%$, and previous urinary tract infection in $11.4 \%$ of the studied population. Enterobacteriaceae were the most frequent isolated microorganisms (44.4\%), and 33.3\% had a polimicrobial culture in abscess samples. S. aureus was rarely identified. Main therapeutic approaches were minimally invasive procedures (pigtails, percutaneous drainage or nephrostomy) in 50\% followed by surgical interventions (nephrectomy or surgical debridement) in $\sim 30 \%$. Only $20.5 \%$ of patients were treated exclusively by antibiotics. Minimally invasive procedures were applied progressively after $2001(\mathrm{p}<0.005)$. In this series case-fatality rate was $4.5 \% ; 13.6 \%(n=6)$ developed septic shock. Nephrectomy was performed in 9 cases $(20.5 \%)$. Patients selected for nephrostomy had a lower risk for ICU admission (Odds Ratio 0.083 IC95 0.008-0.911). Renal abscesses are cause of morbidity but had a low case-fatality ratio; the therapeutic approach has changed in recent years favoring at present minimally invasive procedures.

Key words: Renal absceso, perinephric abscess, therapy, percutaneous drainage, nephrectomy.

Palabras clave: Absceso renal, absceso peri-renal, pionefrosis, tratamiento, drenaje percutáneo, nefrectomía.

\section{Introducción}

$\mathrm{L}$ os abscesos renales y peri-renales corresponden a infecciones supuradas que comprometen el riñón y/o el espacio perinefrítico ${ }^{1,2}$. Son infrecuentes pero potencialmente letales, con cifras de mortalidad que en los primeros reportes alcanzaban $\sim 50 \%$, a pesar de un drenaje adecuado ${ }^{2}$.

No existe una presentación clínica característica del absceso renal y se debe sospechar frente a la presencia de síntomas de infección urinaria que no responden a un tratamiento antimicrobiano adecuado. En pacientes diabéticos y ancianos, esta presentación puede ser aún más vaga y el examen físico muchas veces no refleja la gravedad de la infección, por lo que el diagnóstico se retrasa aumentando el riesgo de mala evolución y su letalidad. Es debido a esto que el diagnóstico definitivo se realiza mediante imágenes.

Las características epidemiológicas de esta patología han cambiado durante las últimas décadas. En la época pre-antimicrobiana predominaban los microorganismos grampositivos, asociados a traumatismos ocupacionales, especialmente en el sexo masculino. Hoy en día, estos cuadros son más frecuentes en mujeres, predominando las bacterias gramnegativas, y se deben generalmente a una complicación tardía de una infección urinaria, espe- cialmente si ésta se asocia con la presencia de urolitiasis ${ }^{3}$. Otros factores de riesgo descritos incluyen diabetes mellitus, obstrucción ureteral, reflujo vesico-ureteral, uso de drogas endovenosas e inmunosupresión.

La cirugía y el uso de antimicrobianos son los pilares fundamentales del tratamiento. Durante los últimos años, y debido a los avances en el estudio de imágenes, han surgido como alternativas varias estrategias mínimamente invasoras tales como el drenaje percutáneo, el uso de pigtails o la nefrostomía, que han obviado la necesidad de procedimientos quirúrgicos.

El objetivo de este trabajo es describir la experiencia clínica y terapéutica sobre esta patología acumulada durante 11 años en nuestro centro asistencial.

\section{Pacientes y Métodos}

Mediante registros computacionales disponibles en el Servicio de Estadística, se identificaron casos de pacientes con el diagnóstico de absceso renal y peri-renal, ingresados a diferentes servicios clínicos del Hospital Clínico de la Universidad de Chile entre los años 1996 y 2006. Se revisaron los registros clínicos y se obtuvieron datos demográficos como sexo, edad, síntomas, factores de riesgo, estudio de laboratorio y de imágenes. En los
Hospital Clínico Universidad de Chile, Santiago Departamento de Urología (JFO, OSC, JFM, FMG, DVF) Departamento de Medicina Sección Infectología (AFC)* Facultad de Medicina, Universidad de Chile Estudiantes de Medicina (JFO, AVP, DVF)

Recibido: 4 de agosto de 2008 Aceptado: 4 de mayo de 2009

\section{Correspondencia a:} Alberto Fica Cubillos albertofica@gmail.com

*AFC trabaja actualmente en Hospital Militar de Santiago, Chile. 
casos en que fue posible aislar un microorganismo, ya fuese del absceso o del urocultivo, se registró la información pertinente.

En todos los casos se obtuvo el número de días de estadía, el lugar de hospitalización (sala general, cuidados intermedios o cuidados intensivos) y el tratamiento reali-

Tabla 1. Características clínicas y de laboratorio. Abscesos renales y peri-renales (n: 44). Hospital Clínico Universidad de Chile

$\begin{array}{lr}\text { Características demográficas } & \text { Frecuencia } \\ \text { Edad promedio en años (rango; desviación estándar) } & 49,9(6-81 ; 17,8) \\ \text { Sexo } & \\ \quad \text { Hombres } & 10(22,7 \%) \\ \quad \text { Mujeres } & 34(77,3 \%) \\ \text { Factores asociados } & \\ \text { Litiasis urinaria } & 16(36,4 \%) \\ \text { Diabetes mellitus } & 17(38,6 \%) \\ \text { Infección urinaria previa } & 5(11,4 \%) \\ \text { Uso de antimicrobianos último mes } & 18(40,9 \%) \\ \text { Cirugía o procedimiento urológico (últimos tres meses) } & 4(9,2 \%) \\ \text { Síntomas } & \\ \text { Fiebre } & 31(70,5 \%) \\ \text { Dolor lumbar } & 36(81,8 \%) \\ \text { Síntomas urinarios } & 15(34,1 \%) \\ \text { Hematuria } & 7(15,9 \%)\end{array}$

\section{Parámetros de laboratorio al ingreso}

Leucocitos por $\mathrm{mm}^{3}$

$\begin{array}{lr}<11.000 & 10(24,3 \%) \\ 11.000-16.900 & 15(36,6 \%) \\ 17.000-25.000 & 14(34,2 \%) \\ >25.000 & 2(4,9 \%)\end{array}$

PCR $\mathrm{mg} / \mathrm{L}$

$\begin{array}{lr}<20 & 1(2,9 \%) \\ 20-100 & 3(8,8 \%) \\ >100 & 30(88,2 \%)\end{array}$

Creatininemia $\mathrm{mg} / \mathrm{dl}$

$\begin{array}{lrr}<1,2 & 12 & (48 \%) \\ 1,2-2,8 & 12 & (48 \%) \\ >2,8 & 1 & (4 \%)\end{array}$

Sedimento urinario zado, ya fuese médico (sólo tratamiento antimicrobiano), invasor (definición arbitraria para la instrumentación con pigtail, drenaje percutáneo o nefrostomía) y/o quirúrgico (aseos quirúrgicos o nefrectomía). Se registró la respuesta al tratamiento y la condición al egreso. Cuando fue posible, se realizó un seguimiento hasta un año desde la fecha del alta con el fin registrar los casos de recidiva.

En relación a las complicaciones, se agrupó a los pacientes en cinco grandes categorías: disfunción renal, disfunción hepática, shock séptico, nefrectomía, muerte. Cuando hubo deceso se indagó acerca de su causa. Todos los datos fueron ingresados a una base de datos digital (programa SPSS v12). Para el análisis estadístico se utilizaron pruebas paramétricas o no paramétricas con un nivel de significación $\mathrm{p}<0,05$.

\section{Resultados}

Características de los pacientes. Se identificaron 44 casos en el período bajo estudio. La edad promedio de los pacientes fue de 49,9 años (rango 6-81 años) y sólo dos pacientes tenían menos de 15 años (4,5\%). La mayoría fue observada en mujeres (77,3\%). Entre los factores de riesgo se encontraron: uso previo de antimicrobianos en el último mes (40,9\%), diabetes mellitus $(38,6 \%)$, litiasis urinaria $(36,4 \%)$, infección urinaria previa $(11,4 \%)$ y el antecedente de alguna cirugía o procedimiento urológico durante los últimos 3 meses (9,2\%) (Tabla 1).

Características clínicas y de laboratorio. Los principales síntomas al ingreso fueron dolor lumbar (81,8\%), fiebre $(70,5 \%)$ y síntomas urinarios $(34,1 \%)$ (Tabla 1$)$.

Más de $75 \%$ del total presentó leucocitosis y elevación de la proteína $\mathrm{C}$ reactiva (PCR), y más de la mitad ingresó con elevación de creatininemia (>1,2 mg/dl) (Tabla 1).

Sólo en $72,1 \%$ de los casos se pudo contar con informe del urocultivo disponible en el registro clínico (Tabla 2). El urocultivo fue positivo para algún microorganismo en aproximadamente $60 \%$ de los casos donde éste fue solicitado. En más de la mitad de los casos en los que se aisló un microorganismo $(\mathrm{n}=19)$, éste correspondió a Escherichia coli (Tabla 2). Aproximadamente en 40\% de los casos se identificaron agentes no habituales como Klebsiella pneumoniae, Enterobacter sp, Proteus vulgaris y Enterococcus sp, correspondiendo este último a $10 \%$ del total de microorganismos aislados (Tabla 2).

El informe de cultivo del absceso constaba en el registro clínico solamente en 9 casos, desconociéndose lamentablemente si se solicitó en todos los pacientes. Los agentes más frecuentes fueron E. coli y K. pneumoniae (Tabla 3). Sin embargo, sólo en tres de estos casos (un tercio) hubo concordancia entre el urocultivo y el agente identificado en el absceso. Estos casos correspondieron 
a abscesos asociados a $E$. coli en dos pacientes con urocultivos positivos con resultado polimicrobiano, asociados a Proteus y Enterococus sp, respectivamente. El tercer paciente tuvo un cultivo de absceso positivo a Enterococcus sp, en quién también se tuvo un urocultivo polimicrobiano (Enterococcus sp y Proteus sp).

Tabla 2. Análisis del urocultivo.

Abscesos renales y peri-renales ( $n$ : 44). Hospital Clínico Universidad de Chile

\begin{tabular}{|lr|}
\hline Análisis del urocultivo & Frecuencia \\
\hline Con urocultivo disponible $\mathrm{n} / \mathrm{N}(\%)$ & $32 / 44(72,1 \%)$ \\
\hline Urocultivo positivo sobre el total disponible & $19 / 32(59,4 \%)$ \\
\hline Microorganismo & \\
\hline Escherichia coli & $12(63,2 \%)$ \\
Enterococcus sp & $2(10,5 \%)$ \\
Klebsiella pneumoniae & $2(10,5 \%)$ \\
Enterobacter aggglomerans & $1(5,3 \%)$ \\
Proteus vulgaris & $1(5,3 \%)$ \\
Enterococcus sp + Proteus mirabilis & $1(5,3 \%)$ \\
\hline
\end{tabular}

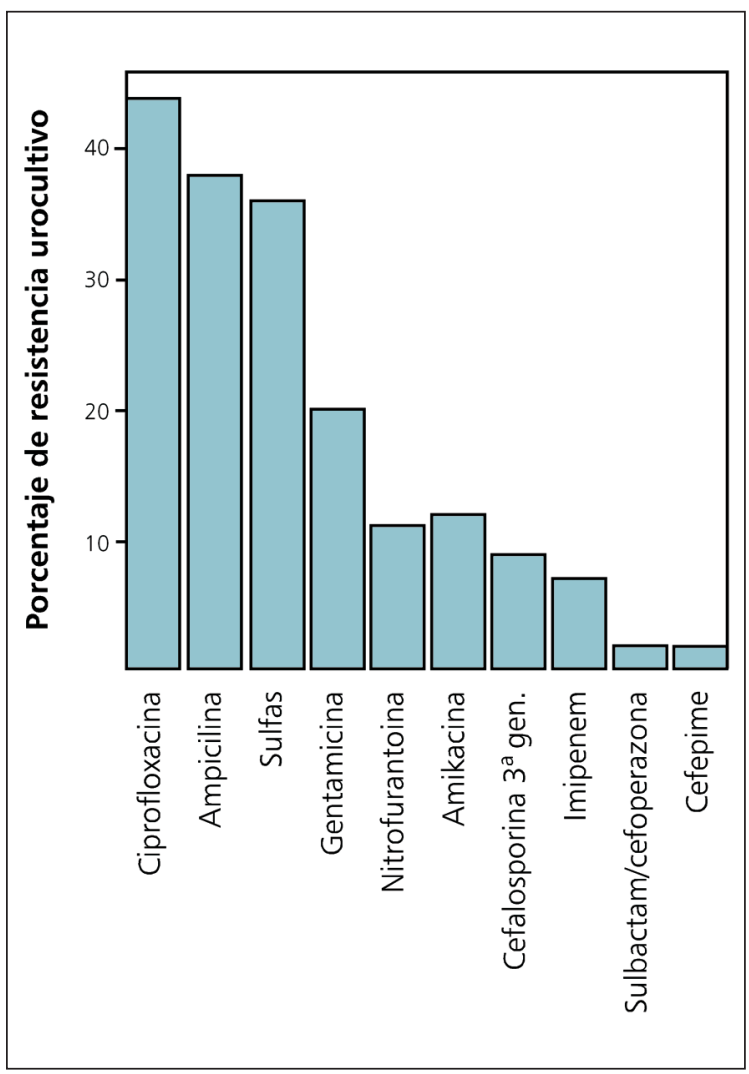

Figura 1. Porcentaje de resistencia a los diferentes antimicrobianos. Abscesos renales y peri-renales (n: 44). Hospital Clínico Universidad de Chile.
En un paciente se identificó S. aureus en el absceso (Tabla 3), asociado a la presencia de un cálculo coraliforme en el riñón, sin evidencias de otro foco del cual pudiese haber provenido el agente. En tres pacientes se identificaron abscesos con cultivos polimicrobianos, dos de ellos con las asociación de enterobacterias-Enterococcus sp, y un caso con E. coli asociada a Fusobacterium sp. En este último caso, la paciente presentó, concomitantemente, una sepsis de foco abdominal por diverticulitis, asumiéndose un mecanismo patogénico de contigüidad.

La resistencia reportada en el urocultivo para los diferentes antimicrobianos se informa en la Figura 1. Destaca el alto porcentaje de resistencia a quinolonas, ampicilina, sulfas, aminoglucósidos e incluso nitrofurantoína y cefalosporinas de $3^{\mathrm{a}}$ generación.

La principal ubicación o tipo correspondió a los abscesos renales $(43,2 \%)$ y en segundo lugar a la pionefrosis $(25 \%)$. La tercera modalidad fue para abscesos perirenales (18,2\%, Tabla 4). No se observó una distribución asimétrica entre abscesos en el lado derecho o izquierdo (Tabla 4).

\begin{tabular}{|lc|}
\hline \multicolumn{2}{|c|}{ Tabla 3. Cultivo del absceso. } \\
Abscesos renales y peri-renales ( $\mathbf{n : ~ 4 4 ) .}$ \\
Hospital Clínico Universidad de Chile
\end{tabular}

Tabla 4. Frecuencia de abscesos renales y peri-renales según su ubicación anatómica (n: 44). Hospital Clínico Universidad de Chile

\begin{tabular}{|lccc|}
\hline Ubicación & Frecuencia & Derecho & Izquierdo \\
\hline Renal & $19(43,2 \%)$ & 13 & 6 \\
Pionefrosis & $11(25,0 \%)$ & 3 & 8 \\
\hline Peri-renal & $8(18,2 \%)$ & $3 *$ & $3 *$ \\
Peri-renal-pionefrosis & $2(4,5 \%)$ & 2 & 2 \\
\hline Otros & $4(9,1 \%)$ & 2 & $19(45,2 \%)$ \\
Total & $44(100 \%)$ & $23(54,8 \%)$ & \\
\hline *2 casos peri-renales sin infomación sobre lateralidad. & & \\
\hline
\end{tabular}




\begin{tabular}{|c|c|c|c|c|}
\hline Ubicación & $\begin{array}{c}\text { Tratamiento médico } \\
\text { solamente } \\
(\%)^{*}\end{array}$ & $\begin{array}{c}\text { Pigtail, drenaje percutáneo } \\
\text { o nefrostomía } \\
(\%)^{*}\end{array}$ & $\begin{array}{l}\text { Aseo quirúrgico o } \\
\text { nefrectomía } \\
(\%)^{*}\end{array}$ & Total \\
\hline Renal & $7(36,8 \%)$ & $6 \quad(31,6 \%)$ & $6(31,6 \%)$ & 19 \\
\hline Peri-renal & $1(12,5 \%)$ & $4 \quad(50,0 \%)$ & $3(37,5 \%)$ & 8 \\
\hline Pionefrosis & $1 \quad(9,1 \%)$ & $7 \quad(63,6 \%)$ & $3(27,3 \%)$ & 11 \\
\hline Peri-renal-pionefrosis & $0 \quad(0,0 \%)$ & $1 \quad(50,0 \%)$ & $1(50,0 \%)$ & 2 \\
\hline Otros & $0 \quad(0,0 \%)$ & $4(100,0 \%)$ & $0 \quad(0,0 \%)$ & 4 \\
\hline Total & $9(20,5 \%)$ & $22 \quad(50,0 \%)$ & $13(29,5 \%)$ & 44 \\
\hline
\end{tabular}

Tabla 6. Tratamiento antimicrobiano usado como primera opción. Abscesos renales y peri-renales (n: 44). Hospital Clínico Universidad de Chile

\begin{tabular}{|c|c|c|}
\hline Antimicrobiano & Frecuencia & Promedio días de uso \\
\hline Cefalosporinas $3^{a}$ gen & $23(54,8 \%)$ & 8 \\
\hline Ciprofloxacina & $12(28,6 \%)$ & 10 \\
\hline Imipenem/cilastatina & $2 \quad(4,8 \%)$ & 9 \\
\hline Cefalosporinas $1^{\mathrm{a}}$ gen & $2 \quad(4,8 \%)$ & 10 \\
\hline Clindamicina & $1 \quad(2,4 \%)$ & 5 \\
\hline Sulbactam/cefoperazona & $1 \quad(2,4 \%)$ & 3 \\
\hline Vancomicina & $1 \quad(2,4 \%)$ & 15 \\
\hline Total & $42 *(100 \%)$ & 8,6 \\
\hline
\end{tabular}

Tabla 7. Forma de resolución según año de ocurrencia. Abscesos renales y peri-renales (n: 44). Hospital Clínico Universidad de Chile

\begin{tabular}{lcccc|} 
Período & $\begin{array}{c}\text { Sólo } \\
\text { antimicrobianos }\end{array}$ & $\begin{array}{c}\text { Técnicas } \\
\text { mínimamente } \\
\text { invasoras }\end{array}$ & $\begin{array}{c}\text { Tratamiento } \\
\text { quirúrgico }\end{array}$ & Total \\
$1996-2001^{*}$ & 6 & 3 & 7 & 16 \\
$2002-2006^{*}$ & 3 & 19 & 6 & 28 \\
Total & 9 & 22 & 13 & 44 \\
Letalidad & - & 2 & - & 2 \\
Recidiva & - & 5 & 1 & 6 \\
\hline
\end{tabular}

${ }^{*} \mathrm{p}<0,005$ por prueba de $\chi^{2}$ para distribución de tipo de tratamiento según período.
Tratamiento. Se analizó en base a la ubicación del absceso y si éste fue llevado a cabo según tres condiciones excluyentes: sólo antimicrobianos, técnicas mínimamente invasoras o cirugía. En términos globales, la principal estrategia terapéutica fue el uso de técnicas mínimamente invasoras $(\mathrm{n}=22 ; 50 \%)$. El aseo quirúrgico $(\mathrm{n}=4)$ o la nefrectomía $(\mathrm{n}=9)$ fueron aplicadas en $29,5 \%$ de los casos. Sólo $20,5 \%(n=9)$ de los pacientes fue sometido a un tratamiento médico exclusivo (Tablas 5 y 6). En orden decreciente, los compuestos más utilizados como antimicrobianos correspondieron a cefalosporinas de $3^{\text {a }}$ generación y quinolonas (Tabla 6). La mayor parte de los pacientes $(n=33 ; 75 \%)$ recibió un tratamiento antimicrobiano combinado y el resto $(n=11 ; 25 \%)$ monoterapia.

Del total de abscesos renales o de ubicación peri-renal, la mayor parte fue manejado con terapia antimicrobiana aislada o procedimientos mínimamente invasores (pigtail, drenaje percutáneo o nefrostomía; 68,4 y $62,5 \%$, respectivamente. El aseo quirúrgico y la nefrectomía fueron utilizados en una fracción menor, cercana al tercio de los casos (Tabla 5).

Es importante señalar que las técnicas mínimamente invasoras presentaron un crecimiento importante como alternativa terapéutica a partir del año 2002. Antes de dicha fecha tan sólo fueron utilizadas en tres oportunidades, aumentando significativamente a 19 casos luego de ese año ( $\mathrm{p}<0,005$ por la prueba de Chi cuadrado, Tabla 7).

De las cinco complicaciones consideradas, la más frecuente correspondió a disfunción renal $(\mathrm{n}=15$; $34,1 \%)$, luego nefrectomía $(\mathrm{n}=9 ; 20,5 \%)$ y shock séptico $(\mathrm{n}=6 ; 13,6 \%)$. En esta serie se reportaron además dos fallecimientos (letalidad 4,5\%), ambos debidos a falla multiorgánica secundaria a shock séptico, observados en el grupo tratado con técnicas mínimamente invasoras (Tabla 7).

La presencia de edad mayor a 60 años, diabetes 
mellitus, litiasis renal o el uso previo de antimicrobianos no se asoció al desarrollo de disfunción hepática o renal, shock séptico o a la necesidad de nefrectomía ni aseos quirúrgicos. Asimismo, el tipo de tratamiento no influyó en el desarrollo de estas complicaciones o en el desenlace fatal que tuvieron algunos pacientes (datos no mostrados). Sin embargo, los pacientes seleccionados para nefrostomía tuvieron menos riesgo de ingresar a UCI respecto a otras modalidades (Odds Ratio 0,083 $\mathrm{IC}_{95}$ 0,008-0,911).

Se observó una tendencia a una mayor estadía hospitalaria según gravedad, utilizando como marcador el lugar de hospitalización. Los tres pacientes ingresados a la Unidad de Cuidados Intensivos tuvieron un promedio de 29 días de estadía ( $\mathrm{DS} \pm 21,9$ ), los pacientes ingresados a la Unidad de Cuidados Intermedios $(n=30)$ tuvieron un promedio de hospitalización de 16,3 días ( $\mathrm{DS} \pm 8,8$ ) y los pacientes internados en sala general $(\mathrm{n}=11)$ presentaron una hospitalización promedio de 11,2 días ( $\mathrm{DS} \pm 6$ ); estas diferencias no resultaron estadísticamente significativas al aplicar una prueba no paramétrica.

Se estudió la recidiva hasta un año post alta, la cual fue posible llevar a cabo en 32 pacientes con seguimiento registrado hasta ese momento. De ellos se reportó recidiva en 6 casos $(18,7 \%)$, ocurriendo en dos de ellos durante el primer mes. Las recidivas fueron observadas en pacientes tratados con técnicas mínimamente invasoras o quirúrgicas (Tabla 7).

\section{Discusión}

El diagnóstico de un absceso renal y peri-renal, tanto en adultos como niños, se hace dificultoso dada su vaga sintomatología ${ }^{2-4-13}$. Se manifiestan principalmente con fiebre, calofríos y dolor lumbar. El examen físico generalmente no refleja la gravedad de la infección por lo que el diagnóstico se retrasa aumentando el riesgo de una evolución desfavorable y la letalidad.

Además de la sospecha clínica es importante la confirmación mediante imágenes, siendo la TAC el examen de elección ${ }^{5}$. Un tratamiento efectivo incluye el uso de antimicrobianos orales y endovenosos.

Los factores asociados con una pobre respuesta al tratamiento antimicrobiano son: abscesos de gran tamaño, uropatía obstructiva, reflujo vésico-ureteral grave, diabetes mellitus, edad avanzada e infecciones por microorganismos formadores de gas. El drenaje quirúrgico se debe considerar cuando no existe una mejoría clínica dentro de los tres primeros días de tratamiento. En el caso que exista uropatía obstructiva se debe descomprimir la vía urinaria lo más rápidamente posible. La nefrectomía se reserva para los casos en que existe un daño extenso $\mathrm{y}$ difuso del parénquima renal y en aquellos pacientes mayores en quienes la sobrevida depende de un manejo quirúrgico agresivo ${ }^{6}$.
La mayoría de los trabajos chilenos publicados referentes al tema, datan de fines de la década de los ochenta por lo que encontramos necesario publicar nuestra experiencia. La mayor serie nacional fue publicada por Baeza y cols en el año 1988, la que incluyó 55 casos de abscesos peri-renales. ${ }^{3}$ Dentro de los resultados, se encontró un claro predominio en el sexo femenino, afectando más frecuentemente al grupo etario entre la cuarta y la sexta década de vida. Los síntomas predominantes fueron dolor, fiebre y compromiso del estado general. Como patologías asociadas, las más frecuentemente encontradas fueron litiasis renal (45\%) y diabetes mellitus $(21,8 \%)$. Dichos resultados son similares a los presentados por Prieto y cols en 1985, quienes describieron un claro predominio en mujeres, mayores de 50 años, siendo el dolor, masa palpable y fiebre los principales síntomas ${ }^{7}$.

En ambos trabajos los principales agentes aislados desde el absceso correspondieron a E. coli, Klebsiella sp, Proteus sp y S. aureus. Como tratamiento, la mayor parte fue sometida a lumbotomía y drenaje quirúrgico. El drenaje percutáneo fue utilizado en una minoría.

Los datos anteriormente expuestos difieren en algunos aspectos a los reportados en nuestra serie. Si bien la epidemiología es similar, manteniendo un claro predominio en el sexo femenino y afectando al grupo etario entre 40 y 60 años, las patologías asociadas han cambiado. La diabetes mellitus se presenta como el mayor factor asociado, observándose en $38,6 \%$ de los pacientes, debiéndose este aumento a una mayor incidencia de dicha patología crónica en nuestra población. La microbiología también ha experimentado modificaciones; si bien E. coli sigue siendo el microorganismo más frecuentemente aislado, $S$. aureus ya no es encontrado de forma frecuente y en nuestra serie solamente fue reportado en un caso. Dicho cambio puede ser explicado ya que antiguamente existía un predominio de este agente en los hombres debido a una mayor incidencia de trauma ocupacional, correspondiendo por lo tanto, la principal vía a diseminación por contigüidad. Actualmente, la mayoría de los microorganismos se diseminan de forma ascendente, predominando aquellos encontrados en la vía urinaria como los bacilos gramnegativos. En el caso de Fusobacterium, su presencia puede ser atribuida a la concomitancia de un proceso diverticular complicado con diseminación del agente al riñón del paciente.

Las complicaciones fueron frecuentes en nuestra serie, destacando una letalidad de 4,5\%, la disfunción renal, el shock séptico y la necesidad de nefrectomía en $20 \%$ de los casos. En esta serie, las dos muertes, secundarias a un shock séptico, correspondieron a pacientes mayores de 65 años y portadores de diabetes mellitus. En ambos casos se trató de un absceso de ubicación peri-renal.

Desafortunadamente, no todos estos pacientes contaron con un estudio microbiológico óptimo, careciendo casi un 
tercio de ellos de un urocultivo para el estudio de esta patología. Además, otro $30 \%$ se presentó con un urocultivo negativo, determinando ambos factores, la ausencia de información necesaria para poder enfocar adecuadamente la antibioterapia. Por otra parte, se pudo determinar que la resistencia de los agentes identificados en esta serie es mayor en porcentaje a lo descrito en pacientes ambulatorios en Chile, destacando el alto porcentaje de resistencia a ciprofloxacina, superior al $40 \%$, cifra ostensiblemente mayor a lo reportado en pacientes no hospitalizados. ${ }^{8-9}$ Las altas tasas de resistencia y la alta frecuencia de agentes inhabituales (sólo $63 \%$ asociada a E. coli, Tabla 2 ), no son sorprendentes en la población afectada por esta patología, que incluye el uso previo de antimicrobianos y antecedentes de infección urinaria (Tabla 1). Por otra parte, el cultivo del absceso demuestra, en ocasiones, una patología polimicrobiana no reconocible desde el urocultivo o que implica otros mecanismos patogénicos como en el caso de Fusobacterium. Todos estos casos señalan las complejidades de la selección de una terapia antimicrobiana inicial actual en estos pacientes.

Otra diferencia importante respecto a otras series nacionales, es el claro predominio de técnicas mínimamente invasoras en lugar de la intervención quirúrgica ( $57 \%$ versus $\sim 30 \%$ ) y el mayor uso de estas alternativas terapéuticas en los últimos años ${ }^{10-11}$. Este hecho refleja los avances tecnológicos y la masificación de estos procedimientos, llevados a cabo, la mayoría de las veces, por radiólogos intervencionales con excelentes resultados. La asociación entre nefrostomía y un menor riesgo de ingreso a Unidad de Cuidados Intensivos descrita en este trabajo, no indica necesariamente una mayor ventaja del procedimiento, sino que puede explicarse como una selección de pacientes menos graves.

Nuestro trabajo tiene varias limitaciones, incluyendo su naturaleza retrospectiva que impide rescatar la totalidad de la información clínica relevante (como por ejemplo datos precisos sobre el momento de la toma del urocultivo -antes o después del inicio de los antimicrobianos- o asociación con bacteriemias- las que no aparecieron como antecedente de la ficha clínica en caso alguno- y con variaciones en las técnicas para el procesamiento de los hemocultivos durante los 11 años del estudio). Asimismo, no existió una estandarización en los procedimientos diagnósticos y en las opciones terapéuticas, el bajo tamaño de la muestra impide encontrar asociaciones significativas y no se pudo contar con datos sobre el nivel de control o daño tisular asociado a diabetes mellitus como factor de riesgo. No obstante, da cuenta de la morbilidad y letalidad asociada a esta condición y de los cambios terapéuticos asumidos en los últimos años. Tampoco es posible, por el diseño descriptivo de este trabajo, contestar la interrogante de si los procedimientos mínimamente invasores influyeron en la letalidad o prolongación de la hospitalización, ya que no estuvieron disponibles en forma homogénea desde el comienzo del período bajo análisis y no fueron randomizados como opción terapéutica.

El diagnóstico de los abscesos renales y peri-renales sigue siendo un desafío para la medicina. Sus características epidemiológicas, las enfermedades asociadas, su etiología y la resistencia antimicrobiana han cambiado en los últimos años ${ }^{12}$. Aunque se ha modernizado su enfoque terapéutico, aún mantiene una carga de morbilidad importante y ocasionalmente es capaz de provocar la muerte.

\section{Resumen}

Los abscesos renales son eventos infrecuentes pero potencialmente letales. Objetivo: Conocer sus características clínicas, diagnóstico y evolución. Metodología: Se efectuó un trabajo descriptivo-retrospectivo con los casos detectados entre 1996 y el 2006 en un centro universitario. Resultados: Se identificaron 44 pacientes (edad promedio 49,9 años) asociados en algunos casos a diabetes mellitus $(38,6 \%)$, litiasis urinaria $(36,4 \%)$ o infección urinaria previa $(11,4 \%)$. Los microorganismos más frecuentes fueron Enterobacteriaceae $(44,4 \%)$ y $33,3 \%$ de los cultivos fueron polimicrobianos. Staphylococcus aureus se identificó infrecuentemente. La estrategia terapéutica principal fue el uso de técnicas mínimamente invasoras (pigtails, drenaje percutáneo o nefrostomía; 50\%), y luego quirúrgicas (nefrectomía o aseos quirúrgicos; 30\%). Sólo $20,5 \%$ fue tratado exclusivamente con antimicrobianos. Los procedimientos mínimamente invasores se usaron en forma progresiva después del 2001 ( $p<0,005)$. La letalidad en esta serie fue 4,5\% $(\mathrm{n}=2)$ y $13,6 \%(\mathrm{n}=$ 6) desarrolló shock séptico. La nefrectomía se aplicó en 9 casos $(20,5 \%)$. Los pacientes seleccionados para nefrostomía tuvieron menos riesgo de ingresar a UCI (Odds Ratio 0,083 IC $_{95}$ 0,008-0,911). Conclusiones: Los abscesos renales son causa de morbilidad mayor aunque de baja letalidad. Su estrategia terapéutica ha ido cambiando en los últimos años a favor de procedimientos mínimamente invasores como los drenajes percutáneos y/o endoscópicos. 


\section{Referencias}

1.- Trucco C, Vilches J, Zambrano N, Domínguez J. Abscesos renales y perinefríticos 28 casos. Rev Chil Urol 1996; 61: 85-9.

2.- Coelho RF, Schneider-Monteiro ED, Mesquita JL, Mazzucchi E, Marmo Lucon A, Srougi M. Renal and perinephric abscesses: Analysis of 65 consecutive cases. World J Surg 2007; 31: 431-6.

3.- Baeza R, Rojas O, Vargas R. Absceso perinefrítico, Análisis de 55 Casos. Rev Chil Urol 1988; 51: 162-5.

4.- Bhat YR. Renal subcapsular abscess. Indian Pediatr 2007; 44: 546-7.

5.- Puvaneswary M, Bisits A Hosken, B. Renal abscess with paranephric extension in a gravid woman: Ultrasound and magnetic resonance imaging findings. Australas Radiol 2005; 49 : 230-2.

6.- Rai C, Karan C, Kayastha BA. Renal and perinephric abscesses revisited. MJAFI 2007; 63: 223-5.

7.- Prieto J, Ramírez H. Abscesos perinefríticos. Rev Chil Urol 1989; 52: 86-8.

8.- Fica A, Pino A, Ramírez P, Brunel V, Triantafilo V. Susceptibilidad ante diferentes antimicrobianos en aislamientos de Escherichia coli de infecciones urinarias ambulatorias en Santiago. Rev Hosp Clinico U Chile 1998; 9: 232-7.

9.- Fica A, Díaz P, López M, Zamorano J, Leiva A, Villalobos C. Altos niveles de resistencia en Escherichia coli causante de infecciones urinarias en una comunidad rural del área metropolitana. Rev Chil Infect 1999; 16: 120-6.

10.- Meng MV, Mario LA, McAninch JW. Current treatment and outcomes of perinephric abscesses. J Urol 2002; 168 : 1337-40.

11.- Shu T, Green J, Orihuela E. Renal and perirenal abscesses in patients with otherwise anatomically normal urinary tracts. J Urol 2004; 172 : 148-50.

12.- Schaeffer A, Schaeffer E. Infections of the Urinary tract. Campbell - Walsh Urology. Ninth edition, 2007. Philadelphia: Saunders Elsevier, pp 273-379.

13.- Dembry L, Andriole V. Renal and perirenal abscesses. Infect Dis Clin North Am 1997; 11: 663-80. 